\title{
Papel importante del alumnado, necesidades psicológicas básicas, regulaciones motivacionales y autoconcepto físico en educación física
}

\author{
Student' important role, basic psychological needs, motivational \\ regulations, and physical self-concept in physical education setting
}

\section{O importante papel dos alunos, necessidades psicológicas básicas, regulações motivadoras e autoconceito em educação física}

\author{
Antonio Méndez-Giménez, Javier Fernández-Río y José Antonio Cecchini Estrada
}

Universidad de Oviedo

\begin{abstract}
Resumen: Se examinó el patrón de relaciones entre la importancia del rol, las necesidades básicas, las regulaciones motivacionales y el autoconcepto físico del alumnado a partir de la teoría de la autodeterminación. Una muestra de 246 estudiantes de 12-17 ańos completó un cuestionario que comprendía las dimensiones papel importante del PMCSQ, autoconcepto físico del DF-AF5, y las escalas BPNES y PLOC. El modelo de ecuaciones estructurales revisado reveló que el papel importante predecía positivamente las necesidades básicas, que a su vez predecían positivamente las regulaciones más autodeterminadas y, negativamente, las menos autodeterminadas. La motivación intrínseca y la regulación introyectada predijeron positivamente el autoconcepto físico. Se sugiere que las intervenciones que mejoran la percepción del alumnado de su importancia en el grupo con independencia de su habilidad, pueden satisfacer sus necesidades y motivaciones y aumentar el autoconcepto físico. Palabras claves: Autoconcepto, autoestima, autodeterminación, Modelo de Vallerand.

Abstract: The Self-Determination Theory was used to examine possible relationships among children's important role, basic needs and motivational regulations. A sample of 246 students, ages ranged from 12-17 years, completed a questionnaire that included the important role subscale of the PMCSQ, the physical self-concept subscale of the DF-AF5, the BPNES, and the PLOC. The structural equation model revealed that the important role positively predicted the basic needs, which positively predicted the
\end{abstract}

more self-determined regulations and negatively the less self-determined ones. Intrinsic motivation and introjected regulation positively predicted physical self-concept too. Results suggest that if teachers can improve students' perceptions of their important role in class, their basic needs and motivations could be satisfied regardless their skill level. They could also increase their physical self-concept.

Key words: Self-concept, self-esteem, self-determination, Vallerand's model. Resumo: Foi examinado o padrăo de relaçóes entre a importância do roll, as necessidades básicas, a regulaçóes motivacionais e o autocontrole físico dos alunos a partir da teoria da autodeterminaçáo. Uma mostra de 246 estudantes de 12 a 17 anos de idade, responderam o questionário que compreendia as dimensóes papel importante do PMCSQ, autoconceito físico do DF-AF5 e as escalas de BPNES e PLOC. O modelo de equaçóes estruturais revisadas mostrou que o papel importante previa positivamente as necessidades básicas, que também previam positivamente as regulaçôes mais autodeterminadas e negativamente as menos autodeterminadas. A motivação intrínseca e a regulação introjetada previram positivamente o autoconceito físico. Sugere-se que as intervençôes que melhoraram a percepçấo dos alunos da sua importância no grupo com independência da habilidade, podem satisfazer as necessidades e as motivaçóes aumentando o autoconceito físico. Palavras chave: autoconceito, autoestima, autodeterminação, modelo de Vallerand.

\section{Introducción}

La participación regular en la actividad física es un componente esencial para determinar el bienestar psicológico y fijar estilos de vida saludables(Jiménez, Martínez, Miró y Sánchez, 2008).En ese sentido, la educación física (EF)constituye un contexto capaz de ejercer una considerable influencia positiva en la salud mental y en la calidad de vida de niños y adolescentes (Standage y Gillison, 2007). Debido a su carác-

\section{Dirección para correspondencia}

Antonio Méndez-Giménez. Departamento de Ciencias de la Educación Universidad de Oviedo (España).Facultad de Formación del Profesorado y Educación. Despacho 219. C/ Aniceto Sela, s/n 33005 Oviedo.

E-mail: mendezantonio@uniovi.es ter curricular obligatorio, esta disciplina es valorada como el vehículo más adecuado para la promoción de estilos de vida saludables entre los escolares (e.g., McKenzie, 2001). Uno de los indicadores de bienestar psicológico más utilizados es el autoconcepto ${ }^{1}$, definido como la medida en que una persona presenta sentimientos positivos generales de su yo (e.g., Balaguer, Castillo y Duda, 2008). La revisión de los estudios sobre intervenciones con niños y adolescentes apoya la hipótesis de que la actividad física produce un aumento en el autoconcepto a corto plazo (Ekeland, Heian y Hagen, 2005). En la

1 En este artículo utilizamos los términos autoestima y autoconcepto como sinónimos. 
actualidad, el autoconcepto se concibe como un constructo multidimensional compuesto por cinco dimensiones: académicollaboral, social, emocional, familiar y físico (e.g., García, Musitu, Riquelme y Riquelme, 2011). Este último, el autoconcepto físico, es especialmente relevante durante la fase de la adolescencia (Fox, 1999).

Hagger, Hein y Chatzisarantis (2011) definen el autoconcepto físico como la opinión global que posee una persona de su propio físico, y refleja el propio juicio de la competencia, condición física y apariencia. El autoconcepto físico se ha relacionado positivamente con la tensión arterial sistólica (Urdampilleta, González, Infante y Goñi, 2011), el rendimiento de los estudiantes en EF (Busso, 2003), la intención y participación en ejercicio físico y actividad deportiva (Esnaola, 2008; Etxaniz, 2005; Infante y Goñi, 2009), la calidad de vida de los adolescentes (Gómez-Vela, Verdugo y GonzálezGil, 2007), y con las formas autodeterminadas de motivación (Thøgersen-Ntoumani y Ntoumanis, 2007; Wilson y Rodgers, 2002).

La Teoría de la Autodeterminación (TAD; Deci y Ryan, 2002) se ha configurado como un marco muy útil para comprenderlos procesos motivacionales, cognitivos y afectivos de los adolescentes tanto en el contexto deportivo como en la EF. Según la TAD, la motivación autónoma debe conducir a la elaboración de índices globales de bienestar (e.g., la autoestima), ya que dicha regulación implica tener una mayor percepción integrada del yo. La TAD sostiene que la motivación autónoma promueve una verdadera autoestima en el individuo, es decir, un sentido estable y seguro de sí mismo (Deci y Ryan, 2000). En esta línea, Hein y Hagger (2007) han encontrado que la motivación autónoma de los alumnos hacia la EF provoca un efecto positivo en la autoestima global informada.

Un primer postulado de la TAD (Deci y Ryan, 2002) sugiere que las motivaciones de un individuo se pueden manifestar a lo largo de un continuum de autodeterminación. En él se describen los siguientes tipos de regulaciones motivacional es: motivación intrinseca, motivación extrínseca (regulación integrada, regulación identificada, regulación introyectada, regulación externa) y desmotivación. La motivación intrinseca se refiere al hecho de realizar una actividad por su interés inherente, ya sea por la excitación o estimulo que permite experimentar, por la satisfacción de explorar y aprender (conocimiento), o por el placer de superar desafíos y alcanzar objetivos (logro). La regulación integrada supone el nivel más alto de determinación dentro de la motivación extrínseca; sin embargo,ha mostrado problemas de medida, especialmente, con sujetos adolescentes. Por este motivo fue excluida en la presente investigación. En la regulación identificada, el individuo efectúa una actividad que no es interesante per se, pero que considera importante porque le permite alcanzar determinadas metas personales. Por ejemplo, un estudiante puede estar motiva- do en la clase EF porque para él es importante hacer bien la tarea. La regulación introyectada representa una forma de motivación relativamente controlada en la que la conducta es regulada por presiones internas para evitar culpabilidad o vergüenza, o para obtener sentimientos de autoestima contingente, aumentar el ego o el orgullo (Deci y Ryan, 2002). Por ejemplo, un alumno puede participar en EF porque se sentiría mal consigo mismo si no lo hiciera. La regulación externa es de naturaleza instrumental, no es más que un medio para lograr otras finalidades. El individuo hace una actividad porque su motivación es controlada por un sistema de recompensas, castigos o coacciones. Por ejemplo, un estudiante puede participar en EF porque se siente obligado a hacerlo o para que el profesor no le rińa. Finalmente, la desmotivación se refiere a los comportamientos que no son motivados ni intrínseca ni extrínsecamente, y que se sitúan en el nivel más débil del continuum de autodeterminación. Los alumnos desmotivados pueden no llegar a entender por qué tienen que realizar clase de EF. Esta situación generalmente sucede cuando los individuos experimentan sentimientos de incompetencia y de falta de control (Deci y Ryan, 2002).

Un segundo postulado de la TAD enfatiza la existencia de tres necesidades psicológicas básicas esenciales para el crecimiento psicológico, el desarrollo social y el bienestar de las personas: autonomía, competencia y relación. La autonomía se define como la necesidad de sentirse el origen y regulador de la propia conducta. La competencia se refiere a la necesidad de producir los resultados deseados y de experimentar maestría y eficacia. Y la relación alude a la necesidad de sentirse capaz de relacionarse con los demás de manera segura y conectada en el contexto social (Deci y Ryan, 2002).Las personas muestran mayor motivación intrínseca si satisfacen dichas necesidades (si perciben libertad de decisión y actuación, si son eficaces en las tareas que desempeńan y si poseen una buena relación con los demás). Sin embargo, si estas necesidades no se satisfacen es probable que el individuo esté motivado de forma extrínseca o desmotivado (Deci y Ryan, 2000).

El tercer postulado de la TAD apunta que determinadas características del contexto social facilitan o constriñen la percepción de satisfacción de esas necesidades (Moreno, Hernández y González-Cutre, 2009).La investigación realizada en el ámbito deportivo y de la EF ha examinado aspectos relevantes del contexto social que predicen la satisfacción de las necesidades, como el clima motivacional. Bajo el paraguas de la Teoría de Metas de Logro, Ames (1992) identificó dos tipos de clima motivacional, el clima de implicación a la tarea o clima de maestría, y el clima de implicación al ego, también llamado clima competitivo o de ejecución. El primero se caracteriza por un ambiente de clase en el que los estudiantes perciben que son recompensados por la mejora personal y el aprendizaje, mientras que en el clima de implicación al ego los 
estudiantes perciben que solo se valora a los más habilidosos. En general, la investigación en el contexto deportivo apoya la idea de que ambos climas tienen un impacto diferente sobre la motivación. En concreto, las percepciones de un clima de maestría se asocian a patrones cognitivos y afectivos adaptativos, como el aumento de la competencia percibida, la diversión, el esfuerzo o el autoconcepto. Por el contrario, las percepciones de un clima de ego se asocian a patrones motivacionales desadaptativos, como el incremento de la preocupación o la orientación a la competición (Cecchini, González, Carmona y Contreras, 2004). En el contexto de la EF, la investigación ha mostrado que la percepción de un clima de maestría se relaciona positivamente con actitudes positivas hacia las clases, alto nivel de interés intrínseco, intención de realizar actividad física y baja ansiedad, mientras que el clima de ego o no se relaciona con la motivación intrínseca o lo hace negativamente (Ntoumanis y Biddle, 1999; Papaioannou, 1998).

Un instrumento ampliamente empleado para evaluar la percepción del clima motivacional en el contexto deportivo es el Cuestionario del Clima Motivacional Percibido en el Deporte (Perceived Motivational Climate in Sport Questionnaire - PMCSQ-2), desarrollado en una primera versión por Seifriz, Duda y Chi (1992) y validado más tarde en el estudio de Newton, Duda y Yin (2000). El cuestionario se compone de 33 ítems y comprende seis subdimensiones del clima motivacional agrupadas en dos factores de segundo orden. Estas subdimensiones fueron etiquetadas como aprendizaje cooperativo, papel importante y esfuerzo/mejora (que reflejan el clima de implicación en la tarea) y castigo por errores, reconocimiento desigual y rivalidad intergrupo (que expresan el clima de implicación al ego).

La TAD mantiene la hipótesis de que el clima de maestría promueve sentimientos de competencia, autonomía y relación, y que, éstos a su vez apoyarán la motivación autodeterminada (Ames, 1992). La evidencia en el ámbito de la EF y del deporte juvenil ha relacionado positivamente el clima de maestría con la competencia percibida (Cecchini, et al., 2004; Ferrer-Caja y Weiss, 2000), la autonomía (Ferrer-Caja y Weiss, 2000; Standage, Duda y Ntoumanis, 2003), y la relación (Sarrazin, Vallerand, Guillet, Pelletier y Cury, 2002). De acuerdo con las predicciones teóricas, una amplia investigación en el contexto deportivo (Sarrazin et al., 2002) y de la EF (Cox y Williams, 2008; Ntoumanis, 2001, 2005; Standage et al., 2003) ha revelado que una mayor satisfacción de estas tres necesidades se relaciona positivamente con las regulaciones autodeterminadas (motivación intrínseca, regulación identificada) y la regulación introyectada.

Finalmente, la TAD postula que la motivación autodeterminada se asocia con el bienestar psicológico y con diferentes consecuencias adaptativas de naturaleza cognoscitiva, emocional y conductual, como el interés, la diversión y la persistencia (Ryan y Deci, 2000). En este sentido, la inves- tigación en el contexto deportivo ha constatado la relación existente entre los niveles más altos de autodeterminación y el bienestar psicológico (Balaguer et al., 2008). En el ámbito de la EF, varios estudios han comprobado que los estudiantes más autodeterminados informan de intenciones más fuertes de participar a corto plazo y en el futuro en las clases de EF, y son más activos físicamente durante el tiempo libre (Ntoumanis, 2001, 2005; Standage et al., 2003).

Reinboth y Duda (2004) apuntaron que un clima percibido de implicación al ego, en el que se evalúa el éxito en términos de comparación social y se refuerza el ser el mejor, es peligroso en términos de desarrollo de autoestima. Por el contrario, la percepción del clima de implicación en la tarea produce con mayor probabilidad un aumento de los niveles de autoestima, teniendo en cuenta que la competencia se concibe de manera autoreferenciada y el éxito se define en términos de dominio, mejora y trabajo duro. Esos factores son relativamente más controlables por la persona. La investigación previa tanto en el contexto deportivo como de la EF ha apoyado la relación entre las percepciones de un clima que implica a la tarea y una mayor autoestima, mientras que las percepciones de un clima que implica al ego han demostrado ser un predictor negativo de la autoestima (e.g. Duda y Kim, 1997).

Apoyándose en los postulados de la TAD, Vallerand (1997) formuló una secuencia de cuatro etapas causales: Factores Sociales $\rightarrow$ Mediadores Psicológicos $\rightarrow$ Tipos de Motivación $\rightarrow$ Consecuencias. Ntoumanis (2001) comprobó empíricamente esta secuencia en el ámbito de la EF evaluando un modelo de relaciones unívocas entre dos subescalas del PMCSQ-2 (aprendizaje cooperativo y esfuerzo/mejora) y una subescala de elección percibida con las necesidades de relación, competencia y autonomía, respectivamente. Posteriormente, varias investigaciones han testado esta secuencia incluyendo ambos climas motivacionales (en su conjunto) como factores sociales o considerando todas las subescalas del PMCSQ-2 (e.g. Cox y Williams, 2008; Méndez-Giménez, Fernández-Río y Cecchini, 2013; Standage et al., 2003). Sin embargo, el papel importante percibido por el alumnado, es decir, la percepción del alumnado de una atmósfera inclusiva en clase, en la que el profesor hace ver que cada estudiante (con independencia de su habilidad, género o capacidad) juega un papel significativo en el grupo, no ha sido todavía estudiado de manera específica en el marco de la TAD. En ese sentido, varios estudios realizados tanto en contextos deportivos como de EF han encontrado correlaciones positivas entre la escala papel importante y la necesidad de relación social (Almagro, SaénzLópez, González-Cutre y Moreno-Murcia, 2011), la competencia percibida (Almagro et al., 2011; González-Cutre, Sicilia y Moreno, 2008) y la autonomía (Méndez-Giménezet al., 2013).

Teniendo en cuenta estos antecedentes, el presente estu- 
dio analiza, desde el marco de la TAD, las relaciones entre el papel importante percibido por los alumnos, las necesidades básicas, los tipos de regulaciones motivacionales y el autoconcepto físico. Para ello, se comprobó un modelo de ecuaciones estructurales basado en la secuencia de Vallerand (1997), que fue formulado de la siguiente manera: Papel importante percibido $\rightarrow$ Satisfacción de competencia, autonomía y relación $\rightarrow$ Tipos de regulaciones motivacionales $\rightarrow$ Autoconcepto físico. Se formularon las siguientes hipótesis: a) que el grado en el que los alumnos perciben que el profesor les confiere un rol importante se relacionaría positivamente con la satisfacción de las tres necesidades psicológicas, b) que éstas predecirían positivamente las regulaciones motivacionales más autodeterminadas (motivación intrínseca y regulación identificada) y la regulación introyectada, y negativamente las menos autodetermiandas, y finalmente, c) que estos tres tipos de motivación más autodeterminadas, a su vez, predecirían positivamente el autoconcepto físico (Fig. 1).

\section{Método}

\section{Participantes}

La muestra estuvo formada por 246 estudiantes de un instituto de enseñanza secundaria del norte de España. De ellos, 132 eran varones y 114 mujeres, lo que constituía el $53.6 \%$ y el $46.3 \%$ de la muestra, respectivamente. Los sujetos eran estudiantes de $1^{\circ}$ de ESO a $1^{\circ}$ de Bachillerato. Las edades oscilaban entre los 12-17 ańos $(M=14.46, D T=1.68)$.

\section{Procedimiento}

En primer lugar, se obtuvo el permiso del Comité de Ética de la Universidad donde se realizó el estudio, así como del centro educativo participante. Posteriormente, se obtuvo consentimiento informado de todos los padres del alumnado participante. Los estudiantes completaron de forma anónima un cuestionario empleando unos 25 minutos de una sesión. Se animó a los estudiantes a que contestaran lo más verazmente posible y se les aseguró que sus respuestas no afectarían a sus notas de EF. Los datos fueron introducidos y analizados mediante el programa informático SPSS, 19.0.

\section{Instrumentos}

Papel importante del alumno. Se empleó la subescala específica del PMCSQ-2 de Newton et al. (2000) en su versión validada y adaptada al contexto español de EF por GonzálezCutre et al. (2008). Esta subescala se compone de 5 ítems, e. g., En mis clases de EF... "cada alumno tiene una función importante", o "el profesor cree que todos nosotros somos importantes para el éxito del equipo/grupo". En el trabajo de González-Cutre el al. (2008) el análisis de la consistencia interna reveló un valor alfa de Cronbach= .70 .

Necesidades psicológicas básicas. Se utilizó la versión validada al español y adaptada a la EF por Moreno, GonzálezCutre, Chillón y Parra (2008) de la Escala de las Necesidades Psicológicas Básicas en el Ejercicio (BPNES) diseñada por Vlachopoulos y Michailidou (2006). El cuestionario consta de 12 ítems agrupados en tres factores (cuatro por dimensión): autonomía (e. g "Tengo la oportunidad de elegir cómo realizar los ejercicios"), competencia (e.g. "Realizo los ejercicios eficazmente") y relación con los demás (e.g. "Me relaciono de forma muy amistosa con el resto de compañeros/ as"). La raíz previa era "En mis clases de EF...”. En la escala original, los coeficientes alpha Cronbach fueron .71, .69 y .84, para la autonomía, competencia, y relación, respectivamente.

Regulaciones motivacionales. Al objeto de determinar los tipos de motivación de los estudiantes de EF se empleó la escala Perceived Locus of Causality (PLOC; Goudas, Biddle y Fox, 1994). La escala fue traducida al espańol y validada para el contexto de la EF en España por Moreno, GonzálezCutre y Chillón (2009). En ese trabajo, los coeficientes alpha de Cronbach para cada una de las subescalas fueron .76 para desmotivación, .70 para regulación externa, .61 para la regulación introyectada, .74 para la regulación identificada, y .75 para la motivación intrínseca, mostrando una consistencia interna aceptable.

Autoconcepto Físico. Se empleó la escala de autoconcepto físico del Cuestionario de Autoconcepto Forma 5 (AF5) (García y Musitu, 1999; García et al., 2011), que consta de 6 ítems específicos y hace referencia a la percepción que tiene el sujeto de su aspecto físico y de su condición física (e. g., "me considero elegante", "soy bueno haciendo deporte"). Los coeficientes alpha de Cronbach para el autoconcepto físico en el estudio de García et al. (2011) fueron de .86 y .87 para las muestras de jóvenes española y chilena, respectivamente. Todos los instrumentos que configuraron el cuestionario de esta investigación emplearon una escala Likert de 5 puntos que oscilaba entre 1 (totalmente en desacuerdo) y 5 (totalmente de acuerdo).

\section{Resultados}

\section{Análisis descriptivos, alphas de Cronbach y correlaciones bivariadas}

La Tabla 1 presenta las medias, desviaciones típicas, alphas de Cronbach y correlaciones para las variables utilizadas en este estudio. Los valores más altos los encontramos en la subescala relación y en la regulación identificada, mientras que los más bajos se observan en la desmotivación y en la regulación externa. Los alphas de Cronbach son, en todas las subescalas, superiores a .70, lo que da muestra de su validez. 
Tabla 1. Alphas de Cronbach, medias, desviaciones típicas y correlaciones entre todas las variables.

\begin{tabular}{lllllllllllll}
\hline & $\alpha$ & $\mathrm{M}$ & $\mathrm{SD}$ & 1 & 2 & 3 & 4 & 5 & 6 & 7 & 8 & 9 \\
\hline 1. P. importante & .75 & 3.77 & .75 & 1 & & & & & & & & \\
2. Relación & .80 & 4.03 & .75 & $.20^{* *}$ & 1 & & & & & & & \\
3. Competencia & .72 & 3.82 & .70 & $.23^{* *}$ & $.40^{* *}$ & 1 & & & & & & \\
4. Autonomía & .73 & 3.27 & .82 & $.34^{* *}$ & $.34^{* *}$ & $.54^{* *}$ & 1 & & & & & \\
5. M. intrínseca & .80 & 3.92 & .80 & $.38^{* *}$ & $.39^{* *}$ & $.65^{* *}$ & $.56^{* *}$ & 1 & & & & \\
6. R. identificada & .83 & 3.99 & .84 & $.41^{* *}$ & $.38^{* *}$ & $.63^{* *}$ & $.51^{* *}$ & $.78^{* *}$ & 1 & & & \\
7. R.introyectada & .70 & 3.22 & .88 & $.25^{* *}$ & .12 & $.32^{* *}$ & $.41^{* *}$ & $.39^{* *}$ & $.41^{* *}$ & 1 & & \\
8. R.externa & .78 & 2.99 & 1.18 & -.07 & -.06 & $-.19^{* *}$ & .01 & $-.25^{* *}$ & $-.15^{*}$ & $.37^{* *}$ & 1 & \\
9. Desmotivación & .82 & 2.02 & 1.01 & $-.33^{* *}$ & $-.19^{* *}$ & $-.36^{* *}$ & $-.14^{*}$ & $-.42^{* *}$ & $-.45^{* *}$ & .09 & $.47^{* *}$ & 1 \\
10. Auto. físico & .79 & 3.71 & .76 & .01 & $.27^{* *}$ & $.50^{* *}$ & $.34^{* *}$ & $.32^{* *}$ & $.31^{* *}$ & $.22^{* *}$ & -.03 & -.06 \\
\hline p<.05 $p<.01$ & & & & & & & & & & & &
\end{tabular}

\section{Análisis de ecuaciones estructurales}

Como se comentó anteriormente, se diseñó un modelo de ecuaciones estructurales en base a las aportaciones teóricas de Vallerand (1997), en el que se hipotetizó que el papel importante asignado por el profesor de EF a sus alumnos predeciría positivamente la satisfacción de las necesidades psicológicas básicas. Sucesivamente se establecieron rutas desde la subes- cala relación a las subescalas motivación intrínseca y regulación identificada, desde la competencia percibida a todos los tipos de motivación, y desde la autonomía a la motivación intrínseca, la regulación externa y la desmotivación. Por último, y en base a las correlaciones observadas en el presente estudio, se establecieron conexiones desde los tipos de motivación más autodeterminados (motivación intrinseca, regulación identificada y regulación introyectada) al autoconcepto físico (Fig. 1).

Figura 1. Modelo de ecuaciones estructurales hipotetizado en base a las aportaciones teóricas de Vallerand (1997).

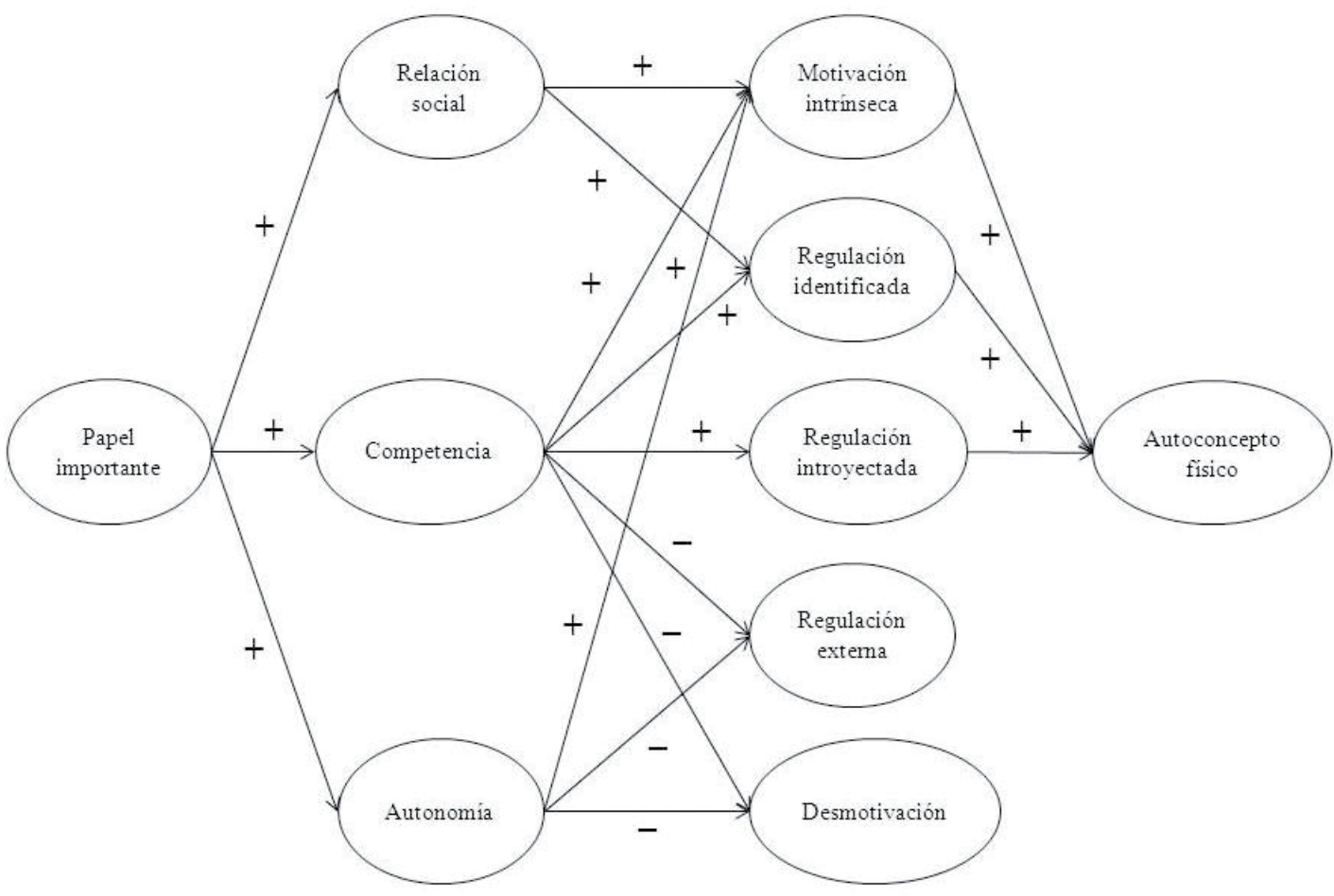


Siguiendo las recomendaciones de Marsh, Richards, Johnson, Rochey Tremayne (1994), todos los análisis factoriales se llevaron a cabo sobre respuestas de pares de ítems en los que los dos primeros elementos de cada escala se promediaron para formar el primer par de ítems, los segundos dos ítems fueron promediados para formar el segundo par de ítems, y así sucesivamente. El uso de pares de ítems es recomendable porque las puntuaciones de pares de ítems son más fiables y contienen menos varianza, ya que la distribución de las respuestas a los pares de ítems tiende a ser distribuida de manera más normal, y porque la relación entre el número de variables medidas y el número de sujetos es la mitad.

Se utilizó el programa AMOS en su versión 18.0. Los resultados mostraron que la estimación normalizada del coeficiente Mardia era relativamente grande (curtosis multivariante $=25.52$ ), por lo que los datos fueron analizados mediante el análisis de máxima verosimilitud robusto junto con el procedimiento de bootstrapping. Este análisis es recomendado por Bentler (1995) cuando los datos no se distribuyen normalmente. Con el fin de evaluar la adecuación del ajuste del modelo propuesto a los datos, se examinaron los siguientes índices de ajuste: el estadístico Chicuadrado, la razón chi cuadrado /grados de libertad ( $\chi^{2} /$ d.f.), el CFI (Comparative Fit Index), el TLI (Tucker-Lewis Index), el RMSEA (Root Mean Square Error Aproximation) y el RMR
(Root Mean Square Residual). En general, un buen ajuste de un modelo específico a los datos se produce cuando la proporción $\chi^{2} /$ d.f. es inferior a 3 (Schermelleh-Engel, Moosbrugger y Muller, 2003), los índices CFI y TLI son superiores a .90 (Huy Bentler, 1995), y el RMSEA (Browne y Cudeck, 1993) y RMR (Hu y Bentler, 1999) son inferiores a .08.

Los índices de ajuste mostraron que la hipótesis del modelo se ajustaba a los datos razonablemente bien aunque había escasas posibilidades de mejora, $\chi^{2}(163)=349.30, p<.001$; $\chi^{2} /$ d.f.=2.14; CFI=.93; TLI=.90; $\quad$ RMSEA=.07; RMR=.05. Considerando las indicaciones de las pruebas de Wald y de los multiplicadores de Lagrange, se realizó un segundo análisis de ecuaciones estructurales para obtener el mejor ajuste posible. Se eliminaron las rutas entre la autonomía y la regulación externa, la autonomía y la desmotivación, y la regulación identificada y el autoconcepto físico puesto que no eran significativas.

El modelo revisado (Figura 2) tuvo un buen ajuste a los datos $\chi^{2}(166)=351.97, p<.001 ; \chi^{2} /$ d.f.=2.12; CFI=.93; TLI=.91; $\mathrm{RMSEA}=.07 ; \mathrm{RMR}=.05$. En la Figura 2 sólo se presenta el modelo estructural (rutas de conexión de factores latentes). El modelo de medición (rutas de conexión de los factores latentes con sus indicadores) ha sido omitido de la Figura 2 para hacer más visuales los datos, no obstante, la información más relevante se presenta en la Tabla 2 .

Figura 2. Modelo revisado del proceso motivacional.

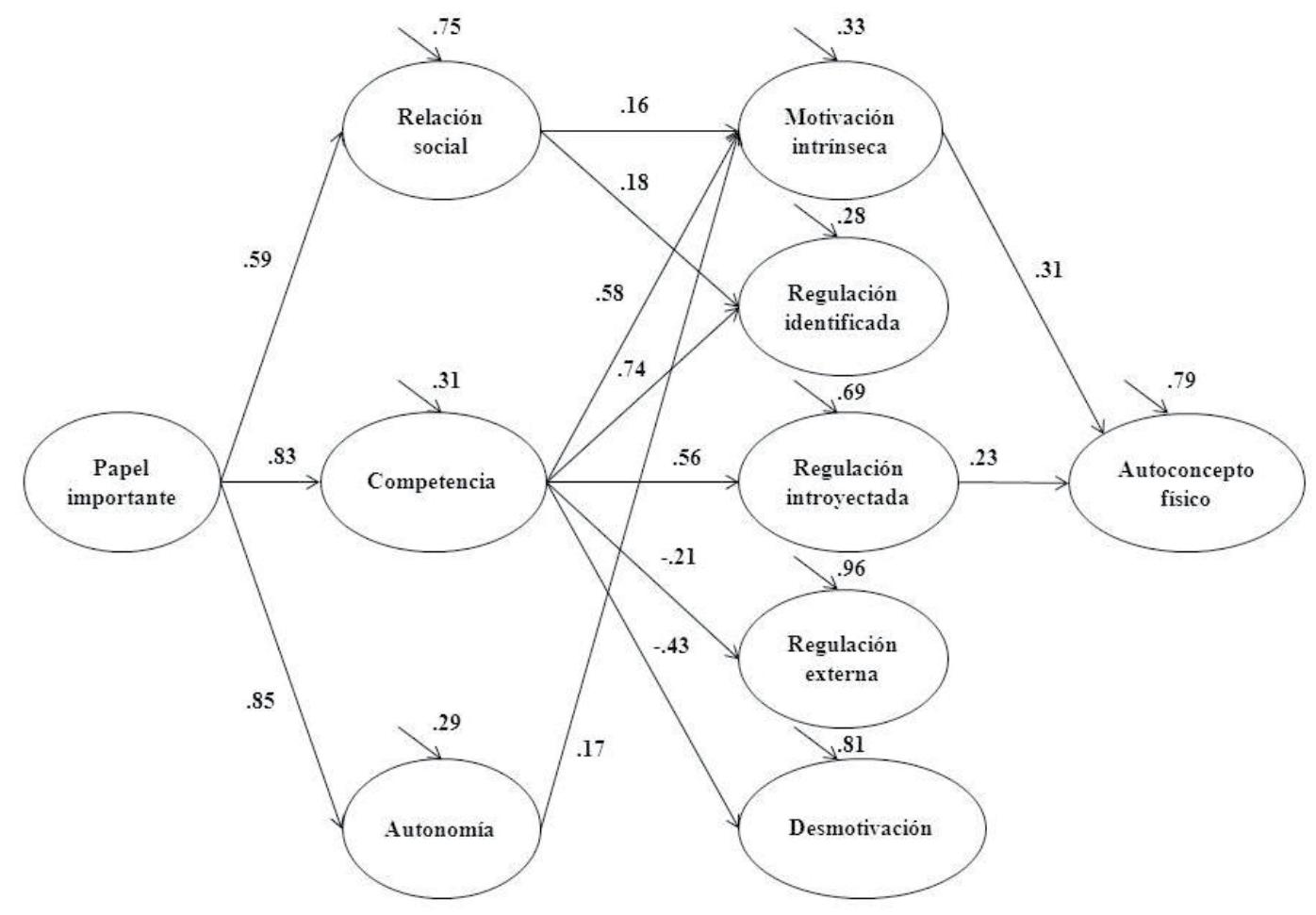


Tabla 2. Carga de los factores y residuos en el modelo de ecuaciones estructurales. Todas las cargas factoriales son significativas al nivel $p<.001$.

\begin{tabular}{lccc}
\hline Factores & Variables & $\begin{array}{c}\text { Carga del } \\
\text { factor }\end{array}$ & Residuos \\
\hline Papel importante & Indicador1 & .827 & .315 \\
& Indicador2 & .510 & .698 \\
Relación social & Indicador1 & .954 & .091 \\
& Indicador2 & .641 & .589 \\
Competencia & Indicador1 & .830 & .322 \\
Autonomía & Indicador2 & .689 & .526 \\
Motivación intrínseca & Indicador1 & .855 & .269 \\
& Indicador2 & .945 & .107 \\
Regulación identificada & Indicador2 & .904 & .182 \\
& Indicador1 & .824 & .491 \\
Regulación introyectada & Indicador2 & .818 & .321 \\
& Indicador1 & .598 & .641 \\
Regulación externa & Indicador2 & .743 & .448 \\
& Indicador1 & .664 & .569 \\
& Indicador2 & .901 & .189 \\
Desmotivación & Indicador1 & .780 & .392 \\
Autoconcepto físico & Indicador2 & .897 & .195 \\
& Indicador1 & .659 & .565 \\
& Indicador2 & .694 & .518 \\
& Indicador3 & .945 & .106 \\
\hline \multirow{2}{*}{ (n) } & & &
\end{tabular}

\section{Discusión}

El principal objetivo de este trabajo era comprobar, en el contexto de la EF, un modelo de ecuaciones estructurales diseńado a partir de los postulados secuenciales del marco teórico de Vallerand (1997) y la TAD. Como factor social se incorporó el constructo papel principal percibido por los alumnos, como mediadores las necesidades psicológicas básicas de autonomía, competencia y relación, en la tercera fase de la secuencia se contemplaron las regulaciones motivacionales y, como consecuencia final, un indicador importante del bienestar psicológico, el autoconcepto físico.

Los resultados obtenidos dan amplio apoyo al modelo revisado. Tal como se hipotetizó, el papel importante predijo positivamente cada una de las necesidades básicas. La relación social se relacionó positivamente con la motivación intrínseca y la regulación identificada. La competencia percibida también se relacionó positivamente con las tres regulaciones más autodeterminadas y, negativamente, con la regulación externa y la desmotivación. Por su parte, la necesidad de autonomía predijo la motivación intrínseca. Finalmente, la motivación intrínseca y la regulación introyectada predijeron positivamente el autoconcepto físico.

\section{Influencia del factor social (papel importante del alumnado) en las necesidades básicas}

Numerosos estudios han demostrado que un clima de implicación a la tarea aumenta la percepción de relación social, competencia y autonomía, tanto en el ámbito deportivo (e.g., Almagro et al., 2011) como en el contexto de la EF (e.g., Cox y Williams, 2008; Ntoumanis, 2001; Standage, Duda y Ntoumanis, 2005). Gran parte de estas investigaciones ha revisado modelos de ecuaciones estructurales en los que el clima de maestría era considerado como un factor de segundo orden explicado por tres subescalas (aprendizaje cooperativo, esfuerzo y papel importante). Este trabajo profundiza en esta evidencia, tomando en consideración exclusivamente el papel importante percibido por el estudiante, que fue integrado en el modelo como único factor social. Las correlaciones bivariadas entre esta dimensión y cada una de las necesidades básicas encontradas en este trabajo y en los preliminares (Almagro et al., 2011; Méndez-Giménez et al.,2013), dan apoyo teórico a la comprobación del modelo. Los resultados del presente estudio avalan la primera hipótesis formulada, reforzando la idea de que un ambiente de clase en el que el docente se esfuerza por enfatizar el papel de todos los alumnos influye positivamente en las percepciones de cada una de las necesidades psicológicas básicas. En primer lugar, se satisface la necesidad de relación. El hecho de que los alumnos de EF perciban que el profesor cuenta con ellos y les otorga un rol relevante, con independencia de su habilidad o género, puede favorecer los lazos de amistad entre compañeros y potenciar una comunicación más fluida. La percepción de un trato igualitario para todos en cuanto a oportunidades de éxito (no solo para los mejores o más dotados) predice un sentimiento de estar conectado con los demás estudiantes y con el profesor. En segundo lugar, el clima generado aumenta la percepción de competencia. Un clima inclusivo favorece la sensación de progreso, eficacia y confianza en el cumplimiento de las exigencias de la clase. En tercer lugar, el énfasis del rol importante de los alumnos con gran probabilidad puede llevarles a percibir un mayor nivel de autonomía, de ajuste con sus intereses y deseos, así como de satisfacer su capacidad de elección y de toma de decisiones.

\section{Necesidades psicológicas y regulaciones motivacionales}

La segunda hipótesis establecía que la necesidad de relación predeciría la motivación intrínseca y la regulación identificada; que la competencia percibida tendría relaciones positivas con las regulaciones más autodeterminadas y, negativas, con la regulación externa y la desmotivación, y que la autonomía 
predeciría positivamente la motivación intrínseca y negativamente la regulación externa y la desmotivación. Nuestros resultados apoyaron completamente las dos primeras relaciones $y$, parcialmente, la tercera parte de esta predicción.

El carácter mediador de las necesidades psicológicas entre el clima de maestría y la motivación intrínseca ha sido mostrado en una gran cantidad de investigaciones realizadas en el contexto de la EF (Ntoumanis, 2001, Standage et al., 2003, 2005). En este sentido, nuestros resultados son congruentes con los estudios citados y, como ellos, revelan el mayor valor predictivo de la necesidad de competencia sobre la motivación intrínseca que las otras dos necesidades. No obstante, satisfacer las relaciones sociales también parece potenciar la motivación intrínseca, la diversión de los alumnos hacia una actividad determinada. Un contexto igualitario generado en clase parece proporcionar oportunidades a los alumnos para satisfacer la necesidad de relación, reforzando los sentimientos de pertenencia y afiliación, lo que también contribuye a aumentar el interés inherente por las actividades realizadas en este contexto (Cecchini et al., 2004; Cecchini, González, Méndez-Giménez y Fernández-Río,2011; Méndez-Giménez et al., 2013). Pero, tal como predice la TAD, este cuadro no sería completo si no se contemplase la mediación de la autonomía sobre la motivación intrínseca. En nuestro trabajo, esta necesidad también aparece como predictora, en contraste con los resultados obtenidos por Ntoumanis (2001), pero en consonancia con estudios posteriores (Ntoumanis, 2005; Standage et al., 2003). Un clima que es percibido como alineado con los intereses del alumnado y que implique cierto grado de libertad para elegir cómo realizar las actividades contribuye igualmente a potenciar el placer intrínseco experimentado en clase de EF.

Tanto la necesidad de competencia como de relación predijeron positivamente la regulación identificada. Es probable que los alumnos que experimentan maestría y eficacia y los que pueden sentirse capaces de relacionarse con los demás en el contexto de la EF estén a su vez más motivados por aprender las habilidades deportivas y quieran hacerlo bien en clase. Estos resultados son consistentes con los obtenidos por Ntoumanis (2001). La conexión entre autonomía percibida y regulación identificada no emergió; sin embargo, esta predicción parece más sólida cuando se contempla el constructo motivación autónoma (motivación intrínseca + regulación identificada, e.g., Standage et al., 2003) o el indice de autodeterminación (Ntoumanis, 2005) en el modelo jerárquico.

Por otro lado, la competencia percibida predijo positivamente la regulación introyectada $y$, negativamente, la regulación externa y la desmotivación. En el algunos casos, los estudiantes pueden sentirse movidos a participar por lo que puedan pensar los demás compańeros o para impresionar al profesor. Al mismo tiempo, como explicó Ntoumanis (2001), los estudiantes con alta percepción de competencia cuentan con menos probabilidades de estar motivados extrínsecamente o desmotivados en las clases de EF. Y viceversa, los que perciben carencias en su habilidad física con mayor probabilidad tendrán dificultades para encontrar sentido a su participación (desmotivados), o lo harán por obligación o temor al castigo (regulación externa).

Finalmente, las dos relaciones entre autonomía y regulación externa y desmotivación fueron eliminadas por falta de significación. Estos datos son, en cierta medida, divergentes de los del estudio de Ntoumanis (2001), quien informó de una predicción negativa entre la autonomía y la regulación externa. Más allá, los resultados del reciente estudio de Lim y Wang (2009), que emplearon una versión modificada del Sport Climate Questionnaire para medir el clima de apoyo de autonomía (escala de 6 ítems), predijeron positivamente la motivación intrínseca y la regulación identificada, y negativamente la regulación externa y desmotivación. Es probable que las diferencias entre estos resultados y los nuestros se deban a la diferente instrumentación empleada para valorar la autonomía percibida y al elevado número de variables incluidas en nuestro modelo.

\section{Regulaciones motivacionales y autoconcepto físico}

La tercera hipótesis pronosticaba que la motivación más autodeterminada (motivación intrínseca, regulaciones identificada e introyectada) actuaría como un predictor positivo del autoconcepto físico, mientras que los tipos de regulación menos autodeterminados (regulación externa y desmotivación) no se relacionarían con el autoconcepto físico. Estas relaciones habían sido formuladas en base a los preceptos de la TAD (Ryan y Deci, 2002), las investigaciones previas sobre el autoconcepto general, los escasos estudios sobre el autoconcepto físico en el contexto del ejercicio y del deporte (ThøgersenNtoumaniyNtoumanis, 2007; Wilson y Rodgers, 2002), y las correlaciones observadas en el presente estudio. Hasta donde sabemos, ningún estudio había profundizado en esta temática en el contexto de la EF.

La relación entre las regulaciones motivacionales y el autoconcepto general ha sido explorada en diversas investigaciones implementadas tanto en el contexto deportivo como de la EF (e. g., Balaguer et al., 2008; Georgiadis, Biddle y Chatzisarantis, 2001; Hein y Hagger, 2007). En el estudio de Georgiadis et al. (2001) la motivación intrínseca y la regulación identificada resultaron predictores positivos del autoconcepto. Los estudios de Balaguer et al. (2008) y Hein y Hagger (2007), para reducir el número de variables en el modelo propuesto y maximizar la parsimonia de los modelos subsecuentes, emplearon un índice de autodeterminación relativo (el RAI-Relative Autonomy Index) calculado mediante la fórmula: motivación intrínseca $(+2)$, regulación identifica$\mathrm{da}(+1)$, regulación introyectada $(-1)$ regulación extrínseca $(-2)$. 
Puesto que la regulación introyectada representa el punto medio del continuo de la autodeterminación no fue considerado en el cálculo del índice de autodeterminación. En ambos trabajos, la motivación autodeterminada emergió como un predictor positivo del autoconcepto. No obstante, el uso de un único índice de autodeterminación no permite explorar el efecto de cada una de las regulaciones motivacionales sobre el autoconcepto.

En cuanto a la relación entre las regulaciones motivaciones y el autoconcepto físico en el ámbito deportivo, pocos estudios han examinado esta cuestión pese a la sugerencia de Fox (1997) de que la autodeterminación puede ser un proceso importante por el cual las personas mejoren su autopercepción física en este contexto. En un trabajo realizado con mujeres deportistas jóvenes, Wilson y Rodgers (2002) encontraron que la motivación autodeterminada hacia el ejercicio (es decir, regulación identificada y la motivación intrínseca) discriminó entre los participantes con alta versus baja autoestima física, mientras que las regulaciones de control del ejercicio (es decir, externa e introyectada) no lo hicieron. En otro estudio, con una muestra diversa de deportistas de clubes deportivos del norte de Inglaterra, Thøgersen-Ntoumani y Ntoumanis (2006) encontraron que la motivación intrínseca predijo significativamente el autoconcepto físico. Controlados por la edad y el sexo, los análisis de regresión múltiple mostraron que la motivación autodeterminada (e.g., la motivación intrínseca y la regulación identificada) predecía patrones de comportamiento, cognitivos y de autoevaluación física más adaptativos que la regulación externa y la desmotivación. Además, la regulación introyectada fue relacionada tanto con resultados adaptativos como desadaptativos. El estudio de Thøgersen-Ntoumani y Ntoumanis (2007), usando la TAD como marco examinó los predictores motivacionales de la preocupación porla imagen corporal, la auto-presentación y las autopercepciones en una muestra de instructores de aerobic. El análisis de regresión reveló que la motivación intrínseca predecía positivamente el autoconcepto físico, y que la regulación introyectada lo predecía negativamente. Los autores discutieron que el ejercicio debido a presiones internas con el fin de lograr autoestima, que depende del tipo de cuerpo ideal definido por la sociedad, puede ser perjudicial para la percepción del yo-físico y para las evaluaciones de la imagen corporal. La autoestima contingente ha sido considerada problemática en varios estudios. Por ejemplo, Patrick, Neighbors y Knee (2004) demostraron que con mayor probabilidad las mujeres que fueron superiores en autoestima contingente se comparan con modelos ideales y experimentan mayores incrementos en la vigilancia y vergüenza corporal.

Nuestros resultados proporcionan apoyo parcial a los argumentos de Fox (1997) y a la investigación previa en el campo deportivo. En el presente estudio, el autoconcepto físico correlacionó positivamente con los tipos de motivación auto- determinada (motivación intrínseca, regulación identificada) y con la regulación introyectada, y no correlacionó ni con la regulación externa y ni con la desmotivación. No obstante, el modelo de ecuaciones estructurales revisado solo reveló que la motivación intrínseca era el principal predictor positivo del autoconcepto físico, seguido de la regulación introyectada. La primera relación es consecuente con el marco de la TAD así como con los resultados de la investigación en el ámbito deportivo. La motivación inherente a la participación en las clases de EF por diversión, por las emociones desencadenadas o el aprendizaje de nuevas habilidades podría provocar un incremento en el autoconcepto físico del alumnado.

Adicionalmente, la regulación introyectada también predijo positivamente dicho constructo de bienestar. Esta relación podría parecer sorprendente y debería ser analizada con más detalle, ya que, de acuerdo con la TAD, se trataría de un tipo motivación controlada que a menudo se asocia con un funcionamiento psicológico desadaptativo (Ryan y Deci, 2000). Sin embargo, esta afirmación ha sido cuestionada por varias investigaciones previas en el contexto de la EF (e.g., Cecchini et al., 2011; Ntoumanis, 2001; Standageetal., 2003), que han encontrado que la regulación introyectada se asocia en ocasiones a variables adaptativas. Por ejemplo, en la investigación de Gillison, Osborn, Standagey Skevington (2009) fue asociada a niveles altos de participación en la actividad física, por encima de los esperados. Además, mientras que la regulación introyectada en los adolescentes varones se relacionó con los factores sociales, como evitar la desaprobación social y la consecución de la mejora del ego, en las adolescentes mujeres reflejó una internalización parcial de las razones para mantener la salud y la condición física. Es posible que la clave esté en la durabilidad de los cambios, pues como mostraron Pelletier, Fortier, Vallerand y Brière (2001), la regulación introyectada fue asociada a la conducta de persistencia a corto plazo, pero no a largo plazo.

Por otro lado, la regulación introyectada es considerada como una forma valiosa de motivación en los casos en que supone el primer paso en el proceso de internalización de la regulación externa hacia metas significativas e importantes a nivel personal (Deci, Eghrari, Patrick, y Leone (1994). Por lo tanto, puede desempeñar un papel fundamental en cómo los adolescentes adoptan actividades y tareas que los profesores de EF les presentan por primera vez, como por ejemplo, aquellas relativas al cuidado y la mejora de la salud (Gillison et al., 2009).

Nuestros hallazgos revelan, pues, que junto con la motivación intrínseca, la presión interna (culpa, vergüenza) que controla el comportamiento de algunos alumnos para tratar de que su profesor piense que son competentes o a para evitar que los compañeros crean que son poco hábiles, también puede producir un incremento del autoconcepto físico. Deci y Ryan (2000) y Kernis (2003) distinguieron entre la 
autoestima verdadera u óptima (es decir, un sentimiento de auto-aceptación más seguro, sólido y estable) y la autoestima contingente (es decir, la que depende de alcanzar algunas metas extrínsecas), y defendieron que sólo el primer tipo de autoestima se relaciona con los procesos de la motivación autodeterminada. Para Deci y Ryan (2000) la autoestima verdadera se desarrolla cuando las personas actúan de manera que satisfacen sus necesidades psicológicas básicas de autonomía, competencia y relación. Las personas con autoestima verdadera muestran un comportamiento intrínsecamente motivado, que es el resultado del interés, diversión, o la lucha por metas significativas, y no de la presión para que coincida con los estándares externos.

Este trabajo proyecta implicaciones evidentes para los educadores físicos. Los resultados ilustran la importancia de enfatizar el rol relevante que juega cada uno de los estudiantes en clase. Este ambiente percibido por los estudiantes potenciaría sus relaciones, les ayudaría a sentirse más hábiles y autónomos, lo que mediatiza el aumento de su motivación autodeterminada. Un niño motivado intrínsecamente tiende con mayor probabilidad a mejorar sus propias valoraciones del yo-físico, aunque también la presión interior ejerce una influencia clave en la configuración de su autoconcepto físico. La repercusión sobre la salud psicológica de los adolescentes podría ser directa, por encima incluso de la influencia de la autoestima global (Sonstroem y Potts, 1996).

Como en todas las investigaciones, este estudio presenta algunas limitaciones. Destacamos dos: su naturaleza trans- versal y correlacional, lo que únicamente permite el ajuste del modelo en un corte determinado de tiempo y no soporta explicaciones de carácter causal. Las investigaciones futuras deberían considerar diseños longitudinales o experimentales, en los que los estudiantes de grupos de edad sean evaluados varias veces durante un período prolongado de participación en clase de EF para determinar la estabilidad de las relaciones a través del tiempo. Además deberían examinar si el autoconcepto físico también puede clasificarse en contingente y verdadero. Las características de este estudio no nos permiten dilucidar esta cuestión, aunque sí identificar dos fuentes o vías predictivas distintas de autoconcepto físico: una derivada de la motivación intrínseca (consecuente con la proposición de autoestima verdadera de Deci y Ryan, 2000) y otra, por mediación de la regulación introyectada (supuestamente de carácter contingente, como apunta la definición de Deci y Ryan, 2002, de este tipo de motivación). Es posible que esta percepción del autoconcepto físico esté condicionada a que el resto de compañeros y el profesor considere que el sujeto en cuestión es atractivo, competente o que cuenta con una buena condición física. En tal caso, esa presión a comportarse para sentirse válido solo provocaría un autoconcepto físico a corto plazo y estaría sujeta a continuas validaciones de sus habilidades o cualidades, como apuntan Vonk y Smit (en prensa). No obstante, en el modelo revisado ambas concepciones de autoconcepto físico parecen confluir en una misma dimensión.

\section{Referencias}

1. Almagro B. J. y Sáenz-López, P. González-Cutre D. y Moreno-Murcia J.A. (2011). Clima motivacional percibido, necesidades psicológicas y motivación intrínseca como predictores del compromiso deportivo en adolescentes, International Journal of Sport Science, 25(7), 250-265.

2. Ames, C. (1992). Achievement Goals, Motivational Climate, and Motivational Processes. En G. C. Roberts (Ed.). Motivation in Sport and Exercise (pp. 161-176).Champaign, Illinois: Human Kinetics.

3. Balaguer, I., Castillo, I. y Duda, J. L. (2008). Apoyo a la autonomía, satisfacción de las necesidades, motivación y bienestar en deportistas de competición: Un análisis de la Teoría de la Autodeterminación. Revista de Psicología del Deporte, 17, 123-139.

4. Bentler, P.M. (1995). EQS structural equations program manual. Encino, CA: Multivariate Software.

5. Biddle S. y Mutrie N. $(2001,2008)\left(2^{\text {nd }}\right.$ Edition). Psychology of Physical Activity: determinants, well-being and interventions. London and New York: Routledge, Taylor and Francis Group.

6. Busso, E. (2003). Aspectos de la habilidad motriz en la educación física escolar. Tesis doctoral inédita. Universidad de Valencia, España.

7. Browne, M. W. y Cudeck, R. (1993). Alternative ways of assessing model fit. En K. A. Bolleny J. S. Long (Eds.), Testing structural equation models (pp. 136-162). Newbury Park, CA: Sage.

8. Cecchini, J. A., González, C., Carmona, A. M. y Contreras, O. (2004). Relaciones entre clima motivacional, la orientación de meta, la motivación intrínseca, la autoconfianza, la ansiedad y el estado de ánimo en jóvenes deportistas. Psicothema, 16, 104-109.
9. Cecchini, J.A. González, C., Méndez-Giménez, A. y Fernández-Río, J. (2011). Achievement goals, social goals, and motivational regulations in physical education settings, Psicothema, 23(1), 51-57.

10. Cox, A. y Williams L. (2008). The roles of perceived teacher support, motivational climate, and psychological need satisfaction in students' physical education motivation. Journal of Sport y Exercise Psychology, 30, 222-239.

11. Deci, E.L., Eghrari, H., Patrick, B.C. y Leone, D.R. (1994). Facilitating internalization: the self-determination theory perspective. Journal of Personality, 62, 119-142.

12. Deci, E.L. y Ryan, R.M. (2000). The "what" and "why" of goal pursuits: Human need and the selfdetermination of behavior. Psychological Inquiry, 11, 227-268.

13. Deci, E.L. y Ryan, R.M. (2002).Overview of self-determination theory: An organismic dialectical perspective. En E.L. Deciy R.M. Ryan (Eds.), Handbook of self-determination research ( $\mathrm{pp}, 3-36)$. University of Rochester Press.

14. Duda, J. L. y Kim, M. S. (1997). Perceptions of the motivational climate, psychological characteristics, and attitudes toward eating among young female gymnasts. Journal of Sports and Exercise Psychology, 19 (Suppl.), S48.

15. Ekeland, E., Heian, F. y Hagen, K. B. (2005). Can exercise improve self esteem in children and young people? A systematic review of randomised controlled trials. British Journal of Sports Medicine, 39.792-798.

16. Etxaniz, I. E. (2005). Autoconcepto físico y satisfacción corporal en 
mujeres adolescentes según el tipo de deporte practicado. Apunts: Educación Física y Deportes, 80, 5-12.

17. Ferrer-Caja, E. y Weiss, M.R. (2000). Predictors of intrinsic motivation among adolescent students in physical education. Research Quarterly for Exercise and Sport, 71, 267-279.

18. Fox, K. R. (1997). The physical self and processes in self-esteem development. En K. R. Fox (Ed.), The physicalself: From motivation to wellbeing (pp. 111-139). Champaign, IL: Human Kinetics.

19. García, F. y Musitu, G. (1999). AF5: Autoconcepto Forma 5. Madrid, España: Tea.

20. García, J.F., Musitu, G. Riquelme, E. y Riquelme, P. (2011).A Confirmatory Factor Analysis of the "Autoconcepto Forma 5" Questionnaire in young adults from Spain and Chile. The Spanish Journal of Psychology, 14(2), 648-658.

21. Georgiadis, M.M., Biddle, S.J.H. y Chatzisarantis, N.L.D. (2001) The mediating role of selfdetermination in the relationship between goal orientations and physical self-worth in Greek exercisers. European Journal of Sport Science, 1, 1-9.

22. Gillison, F., Osborn, M., Standage, M. y Skevington S. (2009).Exploring the experience of introjected regulation for exercise across gender in adolescence. Psychology of Sport and Exercise 10, 309-319.

23. Gómez-Vela, M., Verdugo, M. y González-Gil, F. (2007). Calidad de vida y autoconcepto en adolescentes con necesidades educativas especiales y sin ellas. Infancia y Aprendizaje, 30, 523-536.

24. González-Cutre, D., Sicilia, A. y Moreno, J. A. (2008). Modelo cognitivo-social de la motivación de logro en Educación física. Psicothema, 20(4), 642-651.

25. Goudas, M., Biddle, S. J. H. y Fox, K. (1994). Perceived locus of causality, goal orientations and perceived competence in school physical education classes. British Journal of Educational Psychology, 64, 453-463.

26. Hagger, M.S., Hein, V. y Chatzisarantis, N.L.D. (2011). Achievement goals, physical self-concept and social physique anxiety in a physical activity context. Journal of Applied Social Psychology, 41(6), 1299-1339.

27. Hein, V. y Hagger, M. S. (2007). Global self-esteem, goal achievement orientations, and self-determined regulations in a physical education setting. Journal of Sports Sciences, 15(2), 149-159.

28. Hu, L. y Bentler, P.M. (1995). Evaluating model fit. En R.H. Hoyle (Ed.): Structural equation modeling: Concepts, issues, and applications (pp. 76-99). Thousand Oaks, CA: Sage.

29. Hu, L. y Bentler, P.M. (1999). Cutoff criteria for fit indexes in covariancestructure analysis: Conventional criteria versus new alternatives. Structural Equation Modeling, 6, 1-55.

30. Jiménez, M. G., Martínez P., Miró, E. y Sánchez A. I. (2008). Bienestar psicológico y hábitos saludables: ¿están asociados a la práctica de ejercicio físico? International Journal of Clinical and Health Psychology, 8(1), 185-202.

31. Kernis, M. H. (2003). Optimal self-esteem and authenticity: Separating fantasy from reality. Psychological Inquiry, 14, 83-89.

32. Fox, K. R. (1997). The physical self and processes in self-esteem development. En K. R. Fox (Ed.), The physical self: From motivation to wellbeing (pp. 111-139). Champaign IL: Human Kinetics.

33. Lim, B. S. C. y Wang C. K. J. (2009).Perceived autonomy support, behavioural regulations in physical education and physical activity intention. Psychology of Sport and Exercise, 10, 52-60.

34. Marsh, H. W., Richards, G. E., Johnson, S., Roche, L. y Tremayne, P. (1994). Physical selfdescription questionnaire: Psychometric properties and a multitrait-multimethod analysis of relations to existing instruments. Journal of Sport and Exercise Psychology, 16, 270-305.

35. McKenzie, T.L. (2001). Promoting physical activity in youth: focus on middle school environments, Quest, 53 (3), 326-334.

36. Méndez-Giménez, A., Fernández-Río, J. y Cecchini, J.A. (2013). El Modelo Jerárquico de Vallerand en el contexto de la educación física. Aula Abierta, 41(1), 63-72.
37. Moreno, J. A., González-Cutre, D. y Chillón, M. (2009). Preliminary validation in Spanish of a scale designed to measure motivation in physical education classes: the Perceived Locus of Causality (PLOC) Scale. The Spanish Journal of Psychology, 12(1), 327-337.

38. Moreno, J. A., González-Cutre, D., Chillón, M. y Parra, N.(2008). Adaptación a la educación física de la escala de lasnecesidades psicológicas básicas en el ejercicio. Revista Mexicana de Psicología, 25, 295-303.

39. Newton, M. Duda, J. L. y Yin, Z. (2000). Examination of the psychometric properties of the Perceived Motivational Climate in Sport Questionnaire-2 in a sample of female athletes. Journal of Sports Sciences, $18,275-290$.

40. Ntoumanis, N. (2001). A self-determination approach to the understanding of motivation in physical education. British Journal of Educational Psychology,71, 225-242.

41. Ntoumanis, N. (2005). A prospective study of participation in optional school physical education using a self-determination theory framework. Journal of Educational Psychology, 97, 444-453.

42. Papaioannou, A. (1998). Students' perceptions of the physical education class environment for boys and girls and the perceived motivational climate. Research Quarterly for Exercise and Sport, 69, 267-275.

43. Pelletier, L. G., Fortier, M. S., Vallerand, R. J. y Briere, N. M. (2001). Associations among perceived autonomy support, forms of self-regulation, and persistence: a prospective study. Motivation and Emotion, 25, 279-306.

44. Reinboth, M. y Duda, J. L. (2004). The motivational climate, perceived ability, and athletes' psychological and physical well-being. The Sport Psychologist, 18, 237-251.

45. Ryan, R.M.y Deci, E.L. (2000). Self-determination theory and the facilitation of intrinsic motivation, social development, and well-being. American Psychologist, 55, 68-78.

46. Ryan, R.M. y Deci, E.L. (2002). Overview of Self-Determination Theory: An organismic dialectical perspective. En E.L. Deci, y R.M. Ryan, (Eds.), Handbook of Self-Determination Research (pp. 3-33). Rochester: The University of Rochester Press.

47. Sarrazin, P., Vallerand, R. J., Guillet, E., Pelletier, L. y Cury, F. (2002). Motivation and dropout in female handballers: A 21-month prospective study. European Journal of Social Psychology, 32(3), 395-418.

48. Seifriz, J.J., Duda, J.L. y Chi, L. (1992). The relationship of perceived motivational climate to intrinsic motivation and beliefs about success in basketball. Journal of Sport and Exercise Psychology, 14, 375-391.

49. Schermelleh-Engel, K., Moosbrugger, H. y Müller, H. (2003). Evaluating the fit of structural equation models: Test of significance and descriptive goodness-of-fit measures. Methods of Psychological Research - Online, 8(2), 23-74.

50. Sonstroem, R. J. y Potts, S. A. (1996). Life adjustment correlates of physical self-concepts. Medicine \& Science in Sports and Exercise, 28, 619-625.

51. Standage, M., Duda, J.L. y Ntoumanis, N. (2003). A model of contextual motivation in physical education: Using constructs from self-determination and achievement goal theories to predict physical activity intentions. Journal of Educational Psychology, 95, 97-110.

52. Standage, M., Duda, J.L. y Ntoumanis, N. (2005). A test of self-determination theory in school physical education. The British Journal of Educational Psychology, 75, 411-433.

53. Standage, M., Duda, J.L. y Pensgaard, A.M. (2005). The effect of competitive outcome and task-involving, ego-involving, and cooperative structures on the psychological well-being of individuals engaged in a co-ordination task: A Self-determination Approach. Motivation and Emotion, 29(1), 41-68.

54. Standage M. y Gillison, F. (2007). Students' motivational responses toward school physical education and their relationship to general selfesteem and health-related quality of life. Psychology of Sport and Exercise 8,704-721. 
55. Thøgersen-Ntoumani, C. y Ntoumanis, N. (2007). A Self-determination Theory approach to the study of body image concerns, self-presentation and self-perceptions in a sample of aerobicinstructors. Journal of Health Psychology, 12, 301-215.

56. Urdampilleta, A., González, J., Infante, G. y Gońi A. (2011). La tensión arterial sistémica y el autoconcepto físico en la adolescencia. Cuadernos de Psicología del Deporte, 11(2), 25-33.

57. Vallerand, R. J. (1997). Toward a hier archical model of intrinsic and extrinsic motivation. En M. Zanna (Eds.), Advances in experimental social psychology (pp. 271-360). New York: Academic Press.

58. Vlachopoulos, S. P. y Michailidou, S. (2006). Development and initial validation of a measure of autonomy, competence, and relatedness in exercise: The Basic Psychological Needs in Exercise Scale. Measurement in Physical Education and Exercise Science, 10, 179-201.

59. Vonk, R. y Smit, H. (en prensa). Optimal Self-Esteem is Contingent: Intrinsic Versus Extrinsic and Upward Versus Downward Contingencies. European Journal of Personality. DOI: 10.1002/per.817.

60. Wilson, P.M. y Rodgers, W.M. (2002). The relationship between exercise motives and physical self-esteem in femaleexercise participants: An application of self-determination theory. Journal of Applied Biobehavioral Research, 7, 30-43. 